\title{
Industry update: the latest developments in the field of therapeutic delivery, July 2018
}

\author{
Peter Timmins*,1 \\ ${ }^{1}$ Department of Pharmacy, University of Huddersfield, Queensgate, Huddersfield, HD1 3DH, UK \\ *Author for correspondence: Tel.: +44 148447 3102; p.timmins@hud.ac.uk
}

First draft submitted: 17 August 2018; Accepted for publication: 27 September 2018; Published online: 16 November 2018

\begin{abstract}
Keywords: antisense oligonucleotide $\bullet$ clinical trials $\bullet$ drug delivery $\bullet$ gene therapy $\bullet$ immuno-oncology $\bullet$ mergers and acquisitions $\bullet$ neuroscience $\bullet$ product approvals $\bullet$ rare diseases
\end{abstract}

July 2018, the period covered by this article, appeared to be a good month for industry advances in therapeutic delivery, with strong activity in mergers/acquisitions and collaborations, including gene delivery deals, an oral delivery technology for oligonucleotides and multiple activities in the ever-growing landscape of immuno-oncology. Potential advances in the treatment of rare diseases also figured in the news, and July brought an announcement of the first, long-term, positive data from an investigational HIV vaccine. There was comment in a recent industry update [1] of a reducing investment in neuroscience by industry, and the continued failure in clinical trials of potential treatments for Alzheimer's disease. This month showed that there is in fact much ongoing investment and possibly success in these areas, with work on the discovery of a novel mode of intervention in multiple sclerosis, in possible developments in immuno-neurology, and advances for anti-Alzheimer's drugs - including data on an anti-amyloid $\beta(A \beta)$ antibody that suggest it to be disease modifying.

Information sources employed to provide the materials used in compiling this update included company press releases, conference news and other news websites.

\section{Business news \\ Acquisitions, mergers, licensing \\ Vicore Pharma \& INIM Pharma}

Vicore Pharma Holding AB (Mölndal, Sweden) entered into an agreement with INIM Pharma AB, which is 85\% owned by HealthCap VII LP (Stockholm, Sweden), to acquire INIM. This would afford Vicore access to an INIM candidate and its drug delivery capability in the local treatment of severe interstitial lung diseases such as idiopathic pulmonary fibrosis, and benefit from INIM's collaboration with Nanologica $\mathrm{AB}$ and the latter's technology for enabling poorly water-soluble drugs. Vicore has a novel AT2 receptor agonist for lung disease, C21, which is being readied for Phase IIa in idiopathic pulmonary fibrosis and is being considered for other indications where its antifibrotic activity might offer therapeutic benefit [2].

\section{Axovant Sciences \& Benitec Biopharma}

Exclusive global rights to an investigational gene therapy program from Benitec Biopharma (CA, USA) have been acquired by Axovant Sciences (Basel, Switzerland) [3]. The treatment of oculopharyngeal muscular dystrophy (OPMD), where symptoms including swallowing difficulties, aspiration pneumonia and malnutrition will be explored through the Silence-and-Replace gene delivery technology obtained under the agreement. This technology delivers a combination of DNA-directed RNA (silence) and a functional copy of the faulty gene (replace) in a single adeno-associated viral (AAV) vector construct. Axovant will now advance a preclinical lead, AXO-AAV-OPMD, which targets PAPBN1 and whose mutations led to the muscle cell pathology associated with OPMD. Axovant aims to initiate clinical studies during 2019.

The deal also includes collaboration on five other gene therapy products, the first of these will target C9orf72, which is associated with amyotrophic lateral sclerosis and frontotemporal dementia. 


\section{Otsuka \& Visterra}

A definitive merger agreement has been announced that will lead to the acquisition of Visterra, Inc. (MA, USA) by Otsuka Pharmaceutical Co. Ltd (Tokyo, Japan) [4]. The acquisition brings to Otsuka the Hiertope platform, which combines computational and experimental approaches to design and engineer antibody drug candidates to target highly networked amino acid clusters within an antigen to provide for an antibody with a high affinity [5]. The Visterra pipeline adds programs in IgA nephropathy and other kidney diseases, oncology (multiple myeloma), chronic pain and infectious disease (influenza, dengue fever, Pseudomonas infection) to the Otsuka portfolio.

\section{BioCryst Pharmaceuticals of Idera Pharmaceuticals}

Stockholders of BioCryst Pharmaceuticals (NC, USA) did not approve the merger of the company with Idera Pharmaceuticals, Inc. (MA, USA). That merger would have created a larger rare disease company lead by the CEO of Idera and with the pipeline gain of Idera's TLR9 agonist tilsotolimod. Tilsotolimod is currently in a Phase III trial as an add-on agent to checkpoint inhibitor therapy in the treatment of refractory melanoma. BioCryst will carry on as is, developing small molecule therapeutics for hereditary angioedema (BCX7353), filovirus infection (galidesivir), fibrodysplasia ossificans progressiva (preclinical ALK-2 inhibitor), and has the small molecule influenza therapy peramivir (Rapivab ${ }^{\circledR}$ ) already approved and marketed in several territories [6].

\section{PTC \& Agilis}

PTC Therapeutics, Inc. (NJ, USA), focused on therapies for rare diseases, has entered into an agreement to acquire Agilis Biotherapeutics, Inc. (MA, USA). It has two marketed products, ataluren (Translarna ${ }^{\mathrm{TM}}$ ) approved in the EU, Norway, Iceland, Liechtenstein, Israel and South Korea for the treatment of Duchenne muscular dystrophy caused by a nonsense mutation $(n m D M D)$ of the dystrophin gene, and deflazacort (Emflaza $\left.{ }^{\mathrm{TM}}\right)$, a corticosteroid used in the treatment of Duchenne muscular dystrophy [7]. The acquisition brings AAV vector-based gene therapy development candidates for a number of rare diseases, including the neurotransmitter disease aromatic L-amino acid decarboxylase deficiency with an NDA filing expected in the very near future. Other rare neurological diseases with gene therapy product candidates under development at Agilis include Friedreich's ataxia (preclinical/Phase I) and Angelman syndrome (preclinical) [8].

\section{Genmab \& Immatics}

A strategic partnership to discover and develop next-generation bispecific immunotherapies for multiple oncology indications was announced by Genmab A/S (Copenhagen, Denmark) and Immatics Biotechnologies GmH (Tubingen, Germany) [9]. The deal will enhance Genmab's position in immuno-oncology by bringing together its antibody know-how with Immatics' T-cell receptor capabilities and its XPRESIDENT ${ }^{\circledR}$ target identification high-throughput technology platform. This platform has attomolar detection capability for unique target peptides solely expressed and presented on the cell surface of tumor cells. The deal gives Genmab a license for three targets identified by Immatics with an option to add in two more through additional funding. Genmab will undertake development, manufacturing and worldwide commercialization of any product candidates emerging from the deal; Immatics will have an option to limited co-promotion in selected EU countries.

\section{Kite Pharma \& Gadeta}

Kite, a Gilead Company based in Santa Monica, CA, USA, has entered into a strategic collaboration with Gadeta B.V. (Utrecht, the Netherlands) to develop novel $\gamma \delta$ T-cell receptor immunotherapies for various hematological and solid tumor oncology indications. Gadeta technology involves the engineering of $\alpha \beta$ T cells with $\gamma \delta$ T-cell receptors that then recognize novel targets in cancer cells. Under the deal, Kite will provide the R\&D funding to leverage the $\gamma \delta$ T-cell receptor expertise at Gadeta, which will receive future payments based on milestones achievements. Kite has an exclusive option to acquire Gadeta. The collaboration fits well alongside Kite's existing leadership in engineered T-cell therapies [10].

\section{Dermavant \& GlaxoSmithKline}

The rights to tapinarof, an investigational therapeutic aryl hydrocarbon receptor-modulating agent for the treatment of psoriasis and atopic dermatitis, have been acquired from GlaxoSmithKline (London, UK) by the Roivant subsidiary Dermavant Sciences (AZ, USA) [11], complementing the company's existing pipeline. Tapinarof, applied topically, has completed multiple Phases I and II studies involving over 800 subjects and demonstrated favorable 
clinical outcomes relative to vehicle control. The companies will enter into agreements for supply of investigational drug product for Phase III and for commercial product.

\section{Roche \& PureTech Health}

PureTech Health plc (MA, USA) is engaged in developing innovative medicines based on the crosstalk and biology of the brain-immune-gut axis. The company has announced that it has entered into collaboration with Roche (Basel, Switzerland) to utilize PureTech's milk-derived exosome technology for the oral administration of Roche's antisense oligonucleotide platform. In addition to an upfront payment, Roche will fund research support and milestone payments [12].

Current drug administration approaches cannot utilize natural trafficking systems within the body that transport biologics through the GI tract and into systemic circulation. PureTech's milk-derived exosomes can, and so could enable delivery of oligonucleotides via the oral route to systemic circulation and key tissues, avoiding the issue of poor absorption through intestinal epithelial cells and degradation in the stomach and intestine. Exosomes are involved in intercellular communication and the transport of macromolecules between cells and tissues, and their use as a drug delivery vehicle is attractive as their natural composition may provide superior tolerability over materials used in other current approaches. The technology has evolved out of research undertaken by PureTech and academic collaborators at the University of Louisville (KY, USA) [13].

\section{Novartis \& Galapagos \& MorphoSys}

Novartis (Basel, Switzerland) has acquired the exclusive global development and marketing rights, from Galapagos NV (Mechelen, Belgium) and Morphosys AG (Planegg/Munich, Germany), of MOR106, a monoclonal antibody directed against IL-17C and intended for the treatment of atopic dermatitis [14]. The acquisition adds to the Novartis atopic dermatitis portfolio (they already have an oral administered histamine H4 antagonist, ZPL389 in Phase II trials) and extends their commitment to immuno-dermatology (secukinumab for psoriatic arthritis and ligelizumab for chronic spontaneous urticaria/chronic idiopathic urticaria are both in Phase III trials, and secukinumab is marketed for the treatment of plaque psoriasis).

\section{Sanofi \& Revolution Medicines}

Protein tyrosine phosphatases enzymes regulate critical cellular proteins by selectively removing phosphate groups attached to certain tyrosine residues. One of these, SHP2 (PTPN11), has been shown to regulate the RAS-MAP kinase cell growth signaling pathway whose increased activity is frequently implicated in some human cancers [15]. Sanofi (Paris, France) has licensed RMC-463, the lead small-molecule inhibitor of SHP2 discovered by Revolution Medicines (CA, USA), to develop targeted therapy for patients with non-small-cell lung cancer and other cancers carrying certain mutations. Phase I trials are expected to initiate in the second half of 2018, revolution contributing research and early clinical activity and Sanofi bringing its oncology drug-development experience to later stage activities [16].

\section{Sangamo Therapeutics \& TxCell}

Sangamo Therapeutics, Inc. (CA, USA) and TxCell S A (Valbonne, France) have entered into a definitive agreement by which Sangamo will acquire TxCell. TxCell is engaged in Treg development for immunological disease and the acquisition will enable Sangamo to pursue CAR-Treg therapies in managing solid organ transplant rejection and in autoimmune diseases including Crohn's disease and multiple sclerosis. Proof-of-concept preclinical work has indicated that CAR-Tregs can achieve potent, local immunosuppression, differentiating their action from current immunosuppressants that achieve a global, nonspecific immunosuppression, which could be associated with some of their side effects. Phase I studies are expected to start in 2019 [17].

\section{Biogen \& AliveGen}

Thousand Oaks (CA, USA)-based AliveGen has licensed two investigational drugs that target the muscle growth and function regulator myostatin to Biogen, Inc. (MA, USA). The compounds, ALG-801 in Phase I and preclinical ALG-802, are recombinant proteins that inhibit the myostatin signaling pathway, a differentiated mechanism relative to other studied myostatin approaches, and a mechanism of action that Biogen believes may result in greater efficacy and improved safety. Biogen intends to study the agents in several neuromuscular indications including spinal muscular atrophy and amyotrophic lateral sclerosis [18]. Biogen has already launched modified 
antisense oligonucleotide nusinersen (Spinraza ${ }^{\circledR}$ ) for spinal muscular atrophy and has an investigational agent (BIIB067) for amyotrophic lateral sclerosis in development.

\section{Collaborations \\ AstraZeneca \&o 4D Molecular Therapeutics}

MedImmune (MD, USA), the global biologics R\&D unit of AstraZeneca, and 4D Molecular Therapeutics, also known as 4DMT (CA, USA) have announced their collaboration to develop and commercialize a gene therapeutic for the treatment of chronic lung disease [19]. 4DMT will use its Therapeutic Vector Evolution platform to identify an appropriate carrier to deliver the genetic material to treat the disease. This platform utilizes over 100 million unique AAV variants from over 35 unique and proprietary 4DMT AAV libraries, identifying lead vectors from those libraries resulting in a customized, novel carrier specifically designed to create a medicine for targeted gene delivery in human patients.

\section{Gritstone Oncology \& Bristol-Myers Squibb}

A clinical trial collaboration has been announced by Bristol-Myers Squibb (NY, USA) and Gritstone Oncology (CA, USA) to evaluate the safety and tolerability of Gritstone's personalized neoantigen immunotherapy, GRANITE-001, in combination with BMS's marketed immuno-oncology therapeutics, the PD-1 immune checkpoint inhibitor nivolumab (Opdivo ${ }^{\circledR}$ ) and nivolumab plus ipilimumab (Yervoy ${ }^{\circledR}$ ), a CTLA-4 blocking human monoclonal antibody. GRANITE-001 is dosed as sequential delivery of neoantigens within an adenovirus-based vector (prime) and a self-replicating RNA-based vector (boost) to elicit an antigen-directed T-cell response. The Phase I trial under this collaboration is expected to initiate before the end of 2018, with Gritstone Oncology as the sponsor [20].

\section{Takeda \& Ovid Therapeutics}

Takeda Pharmaceutical Company (Osaka, Japan) and its partner Ovid Therapeutics Inc. (NY, USA) have updated on their clinical plan for TAK-935/OV935 in rare epilepsy conditions, expanding into treatment of younger patients. The companies will initiate three clinical trials, one in children with Dravet syndrome and Lennox-Gastaut syndrome, a second one in pediatric patients with CDKL5 deficiency disorder and Duplication 15q syndrome, and the third trial being an extension trial for patients with developmental and epileptic encephalopathies who had participated in a previous TAK-395/OV935 clinical study [21]. These rare diseases typically present early in life, are often associated with severe cognitive and development problems, and those affected suffer frequent treatmentresistant seizures throughout their lifetime. TAK-935/OV935 is a novel mechanism of action antiepileptic agent, being a first-in-class cholesterol 24-hydroxylase inhibitor. The 24-hydroxycholesterol, formed from cholesterol in the brain by this enzyme, may be involved in overactivation of the glutaminergic pathway. Decreasing its levels by inhibiting cholesterol 24-hydroxylase could decrease glutamate hyperactivity. Glutamate, a major neurotransmitter in the brain, has been shown to play a role in the initiation and spread of seizure activity. The two companies initiated their development collaboration on this Takeda-discovered asset at the beginning of 2017 [22].

\section{Anima Biotech \& Eli Lilly}

A multiyear collaboration has been agreed between Anima Biotech (NJ, USA) and Eli Lilly (IN, USA) for the discovery and development of translation inhibitors of several target proteins using Anima's Translation Control Therapeutics platform. This technology is a new strategy against 'undruggable' target proteins. Such drug targets are challenging because the binding of drug small molecules to proteins implicated in disease to modify their activity is hampered by the accessibility of binding sites. The Anima approach, utilizing a cloud-based platform and employing novel biology, proprietary image analysis and high performance 'big data' software, is to find compounds that work one step before the protein is produced by ribosomes, and modulate that process rather than trying to act on the protein after it has been generated. Anima is working in multiple therapeutic areas including fibrosis, viral infections, oncology and immunology [23].

\section{GlaxoSmithKline of 23andMe}

GlaxoSmithKline and 23andMe (CA, USA) have announced an exclusive 4-year collaboration using human genetics as the basis for discovery of innovative new medicines. The collaboration will combine 23andMe's largescale genetic resources and advanced data science skills with the drug development and commercialization expertise of GlaxoSmithKline. Through data acquired from its customers as part of its genetic profiling service, 23andMe 
owns what is now the world's largest human genetic and phenotypic resource. The collaboration will explore this database to develop improved understanding of how genes impact health and so potentially discover novel drug targets, identify patient subgroups that are more likely to respond to certain therapeutic interventions, and focus on recruitment of the most appropriate patients for clinical studies [24].

\section{Other business news}

Convelo Therapeutics

Convelo Therapeutics (OH, USA) announced its intent to discover and develop a new class of medicines that can regenerate the myelin sheath around nerve cells, which is lost in a number of neurological diseases including multiple sclerosis [25]. Based on work at Case Western Reserve University School of Medicine (OH, USA), Convelo's discovery platform is founded on stimulating the regeneration of new myelinating cells. CNS-penetrating small molecules that stimulate oligodendrocyte differentiation and myelin regeneration from oligodendrocyte precursor cells are being pursued, inspired by observation of this stimulation and the associated generation of 8,9-unsaturated sterols through inhibition of a narrow range of enzymes within cholesterol biosynthesis. This inhibition is seen with certain already known small molecules, including azole antifungal agents [26]. In addition to multiple sclerosis, myelin loss or dysfunction is involved in several rare childhood neurological diseases and in spinal cord injury, stroke and traumatic brain injury.

\section{Alector}

Immuno-oncology is now firmly established, with approved agents for a range of indications and a very significant clinical and preclinical space promising new agents, new combinations of agents and additional indications. Immuno-neurology may be less familiar, but emerging biotechnology company Alector (CA, USA) has that (as well as immuno-oncology) in its sights, focused on developing therapies that harness the immune system to treat neurodegenerative diseases. The company has just announced securing funding, which will be used to advance its early stage pipeline. Alector's approach is to use the immune system in the brain to simultaneously counteract multiple local pathologies, in contrast to existing therapeutic approaches in neurodegenerative diseases that target just one aspect, for example, $\beta$-amyloid or tau. Three candidates are being progressed into clinical development; AL001 for the treatment of frontotemporal dementia, AL002, which acts to increase TREM2 activity (deficiency in TREM2 is associated with early onset dementia and early death) and will be explored in Alzheimer's disease, and AL003, which targets SIGLEC-3, a transmembrane receptor whose expression on myeloid lineage cells is a risk factor for Alzheimer's disease [27].

\section{Inversago Pharma}

Inversago Pharma, Inc. (Montreal, Canada) is developing new-generation cannabinoid-1 (CB-1) receptor blockers to overcome problems seen with first-generation agents. First-generation agents have good efficacy in a range of metabolic diseases, but were able to cross the blood-brain barrier, with significant central CB-1 receptor occupancy. This led to serious psychiatric side effects that resulted in the discontinuation of all clinical research on these agents. Inversago is advancing peripherally restricted CB-1 receptor blockers, which could avoid those centrally mediated adverse events. The central effects were thought to be required for efficacy, but it has now been shown in preclinical studies that peripheral CB-1 blockade could be effective in metabolic disease. Inversago plans to take its new-generation CB-1 blockers into clinical trials across a range of potential metabolic indications, including Prader-Willi syndrome, Type 1 diabetes (blocking CB-1 expressed in pancreatic $\beta$-cells promotes insulin secretion and $\beta$-cell proliferation/survival), obesity and nonalcoholic steatohepatitis (NASH) [28].

\section{Alzheon}

A potential approach to mild Alzheimer's disease in a specific patient group was presented at the Alzheimer's Association International Conference (Chicago, IL, USA, 22-26 July 2018) by Alzheon, Inc. (MA, USA). New analyses of Phase III data, focused on APOE4/4 homozygous patients with mild Alzheimer's disease, in subjects treated with tramiprosate (homotaurine) indicated large clinical benefit on both cognition and function in this patient subgroup at the highest dose of drug. Alzheon are intending to use this analysis to support the advancement of their tramiprosate prodrug ALZ-801, into a pivotal trial [29]. Tramiprosate has been shown to inhibit A $\beta$ monomer aggregation and formation of toxic soluble $A \beta$ oligomers but is challenged by poor tolerability and variable absorption. ALZ-801, the valine prodrug of tramiprosate, is absorbed as the prodrug with metabolism to 
the active form primarily in the liver [30]. It has been shown to have good tolerability and reduced intersubject variability in absorption relative to parent tramiprosate, and a longer apparent elimination half-life [31].

\section{IFM Therapeutics}

IFM Therapeutics LLC (MA, USA) has announced the launch of a subsidiary company, IFM Tre (MA, USA), which is developing NLRP3 antagonists that may block the inflammatory responses that underlie a variety of diseases, including metabolic, fibrotic, autoimmune and neurodegenerative diseases [32]. IFM Tre's programs act on the innate immune system, suppressing only the NRLP3-mediated inflammation, leaving other pathways unsuppressed to avoid impacting normal responses to pathogens. The new company has three current development programs. The lead candidate acts on NRLP3 outside of the CNS, inhibiting the generation of proinflammatory IL-1 and -18. Phase I trials are expected to start in 2019. Two earlier stage candidates are a gut-directed compound that could be used as single agent or as part of combination therapy in inflammatory bowel disease, and a further agent that can cross the blood-brain barrier and may be of value in Alzheimer's disease and other neurodegenerative and neuroinflammatory conditions. Bristol-Myers Squibb had acquired the rights to IFM Therapeutics preclinical NLRP3 agonist program, along with its preclinical stimulator of interferon genes (STING) agonist program last year to add into its immuno-oncology portfolio [33].

\section{Product approvals \& other regulatory news Ivosidenib, Tibsovo ${ }^{\circledR}$, Agios Pharmaceuticals}

A precision medicine approach to the treatment of a specific set of adult patients with relapsed/refractory acute myeloid leukemia (AML) has been approved by the US FDA. Agios Pharmaceuticals, Inc. (MA, USA) has announced the approval of ivosidenib (Tibsovo ${ }^{\circledR}$ ), an oral inhibitor of isocitrate dehydrogenase-1 (IDH1) for the treatment of AML in patients with a demonstrated susceptible IDH1 mutation. The majority of AML patients eventually relapse, and relapsed/refractory AML has a poor prognosis. The availability of ivosidenib represents a new, targeted treatment option for those $6-10 \%$ of AML patients where a mutation of IDH1 is a contributor to their disease. It can offer a unique approach to helping these patients compared with conventional chemotherapy [34].

\section{Elagolix, Orilissa ${ }^{\mathrm{TM}}$, AbbVie}

The FDA approval of the first new oral treatment in a decade for the management of moderate to severe pain associated with endometriosis has been announced by AbbVie (IL, USA). Elagolix (Orilissa ${ }^{\mathrm{TM}}$ ) is an oral gonadotropinreleasing hormone antagonist specifically developed for the treatment of moderate to severe endometriosis pain. Endometriosis affects an estimated $10 \%$ of women of reproductive age and can be associated with debilitating pain symptoms. Current treatment involves medicines not necessarily specifically developed for treatment of endometriosis, such as nonsteroidal anti-inflammatory drugs or opioids, or hormonal treatment including oral contraceptives. In some cases, surgery may be used. Elagolix is a nonpeptide, small-molecule antagonist that binds competitively to gonadotropin-releasing hormone receptors in the pituitary gland, resulting in a dose-dependent luteinizing hormone (LH) and follicle-stimulating hormone (FSH) suppression with a consequent reduction in blood levels of estradiol and progesterone [35]. The compound was originally discovered by Neurocrine Biosciences, Inc. (CA, USA) and is currently also in Phase III trials for the treatment of uterine fibroids with an expected NDA filing in 2019 [36].

\section{Tecovirimat, Tpoxx ${ }^{\circledR}$, SIGA Technologies}

Tecovirimat $\left(\operatorname{Tpoxx}^{\circledR}\right)$ is a small-molecule antiviral for the treatment of smallpox that has been approved by the FDA for use in mitigating the impact of an outbreak. The approval was announced by the drug's developer, SIGA Technologies (NY, USA). Smallpox, although eradicated as a naturally occurring disease, represents a significant bioterrorism threat and the availability of tecovirimat provides a way to reduce the morbidity and mortality associated with an outbreak should such a threat develop. The approach to clinical validation of tecovirimat involved demonstration of efficacy in animal models of smallpox and safety in human clinical trials. A long shelf life of 7 years is assigned to the product and, based on that shelf life determination, an option to exercise a $\$ 50$ million payment by Biomedical Advanced Research and Development Authority to SIGA has been requested under an existing contractual agreement between the two parties. SIGA has delivered 2 million courses of therapy to the US Strategic National Stockpile, which is the only route through which the drug will be made available. SIGA has indicated its intent to evaluate additional indications for tecovirimat and explore additional markets [37]. 


\section{Tafenoquine, Krintafel ${ }^{\mathrm{M}}$, GlaxoSmithKline}

Although the Plasmodium vivax parasite infects the blood after a bite by an infected mosquito and results in an acute malaria episode, it can then sit dormant in the liver, reactivating every so often - weeks, months or even years after the original infection - to cause relapses. Therapies against the blood stage of the parasite are not so effective against its dormant form, with a 14-day course of the 8-aminoquinoline primaquine being the current option. A new option was announced by GlaxoSmithKline and Medicines for Malaria Venture (Geneva, Switzerland), with the FDA approval of a new 8-aminoquinoline, tafenoquine (Krintafe ${ }^{\mathrm{TM}}{ }^{\mathrm{N}}$. Tafenoquine is a single-dose therapy, hence overcoming the potential compliance issues of primaquine, and is used along with available blood stage antimalarial agents to achieve prevention of relapse (radical cure) in cases of acute $P$. vivax infection. Tafenoquine represents the first new treatment for this kind of malaria for decades and is anticipated to contribute to ongoing effort to eradicate the disease [38]. Tafenoquine will be provided as a not-for-profit medicine in malaria-endemic countries to maximize access by those who need it most.

\section{Romosozumab, Evenity ${ }^{\top M}, U C B$, Amgen}

The protein sclerostin, encoded by SOST in osteocytes and some chondrocytes, binds to LRP5/ 6 receptors inhibiting the Wnt signaling pathway and leading to decreased bone formation by osteoblasts. A sclerostin-targeting antibody, romosozumab (Evenity ${ }^{\top \mathrm{T}}$ ), has been in development jointly by Amgen (CA, USA) and UCB (Brussels, Belgium) for the treatment of osteoporosis in postmenopausal women at high risk of fracture. In the USA, half of the women over 50 will suffer a fracture due to osteoporosis and there is a need for improved approaches to management of fracture risk. However, a previous Biologics License Application (BLA) filing was the subject of a complete response letter by the FDA on the basis of a cardiovascular safety signal [39]. The two companies have worked since the complete response letter to prepare a resubmission, with additional data from a further clinical trial to address the risk-to-benefit of the medicine. They have now announced that the resubmission has been made to the FDA. The EMA and the Pharmaceuticals and Medical Devices Agency (PMDA, Japan) are also currently reviewing marketing applications for romosozumab [40].

\section{Clinical trials \\ Ibrexafungerp (SCY-078, Scynexis) in vulvovaginal candidiasis}

Scynexis (NJ, USA) has announced positive results from their Phase IIb study of its novel antifungal agent ibrexafungerp in moderate-to-severe vulvovaginal candidiasis (VVC). Ibrexafungerp is a first-in-class, orally bioavailable $\beta-1-3$ glucan synthase inhibitor that targets the fungal cell wall, resulting in a weakened structure that fails under osmotic stress leading to cell lysis. This distinguishes it from established antifungals (polyenes and azoles), which work by affecting ergosterol pathways in the fungal cell membrane and so it could overcome any emerging resistance to these classes of agents. Although it shares mechanism of action with the echinocandin antifungals, it has the advantage over them of oral bioavailability, therefore offering greater dosing flexibility. Ibrexafungerp emerged out of a collaborative discovery and development effort between Scynexis and Merck (NJ, USA) with all rights subsequently being transferred to Scynexis. An end of Phase II meeting with the FDA is planned, to agree on a development pathway for Phase III trials. Indications in azole-resistant infection are envisaged, including systemic infections where it would offer an alternative to echinocandins for Aspergillosis and as an alternative to azoles in VVC, including hard-to-treat recurrent VVC [41].

\section{Fenfluramine hydrochloride (ZX008, Zogenix) low-dose therapy for Dravet syndrome}

Fenfluramine hydrochloride, a serotonergic/noradrenergic agent previously prescribed as an appetite suppressant, was withdrawn from in most markets in the late 1990s because it was linked to the development of heart valve defects. Zogenix (CA, USA) has explored repurposing of the drug, employing its low doses $(0.2$ and $0.5 \mathrm{mg} / \mathrm{kg})$ administered as an oral solution (ZX008), in children and young adults with Dravet syndrome. In a second Phase III trial, it met all primary and secondary end points in terms of reductions in seizure frequency compared with placebo when added on to standard of care in these patients. The treatment was well tolerated with similar adverse events frequency in active and placebo groups, with no cardiovascular safety issues noted over the course of the study [42]. The new study data adds onto and supports the previously released data from the first Phase III trial [43] and, as a result, Zogenix is planning to file applications for regulatory approvals in the USA and in Europe before the end of 2018. 
TORC1 inhibitor RTB101 (PureTech Health/resTORbio)-positive Phase $2 b$ results in respiratory tract infections in the elderly

resTORbio (MA, USA), an independent affiliate of PureTech Health (noted earlier in this article for their deal with Roche on milk exosome technology), has announced positive Phase IIb data, for its RTB101 as treatment to reduce the risk of morbidity and mortality associated with respiratory tract infections (RTIs) in elderly patients. RBT101 is an oral, selective, potent inhibitor of TORC1. Relative to placebo, it showed a statistically significant and clinically meaningful reduction in the percentage of patients with one or more laboratory-conformed RTIs. Dosing 20 mg RBT101 per day or adding a low dose of TORC1 inhibitor everolimus to RTB101 led to loss of benefit in prevention of RTIs, suggesting that there is an optimum degree of inhibition of TORC1 required. The company indicates that it has a way forward to Phase III, having now defined the dose and identified patient groups that were particularly high responders, and is planning an end of Phase II meeting with the FDA [44].

\section{Clascoterone (Winlevi ${ }^{\mathrm{TM}} /$ Breezula ${ }^{\mathrm{TM}}$, Cassiopea) topical antiandrogen in acne \&o androgenetic alopecia}

The results from two Phase III trials of topical 1\% clascoterone cream have demonstrated statistically significant improvements in the reduction in severity of acne relative to placebo, as detailed in an announcement from Cassiopea Spa (Lainate, Italy). Clascoterone antiandrogen treatment represents a novel mechanism of action in the therapy of acne, working at the top of the cascade of events generating inflammation and lesions. Applied topically, it reaches the androgen receptors in the sebaceous glands and blocks the effect of endogenous androgens, preventing their generation of excess sebum and the development of acne. Clascoterone is then rapidly metabolized to cortexolone, a component of the endogenous corticosteroid pool, with a consequence that it attains high local antiandrogen activity without systemic side effects [45]. Cassiopea is also developing a higher strength (2.5-7.5\%) topical solution formulation of clascoterone for the treatment of androgenic alopecia, which has shown positive Phase II data in terms of increased hair growth relative to placebo control [46].

\section{Anti-AB protofibril antibody BAN2401 (Eisai, Biogen) slows Alzheimer's decline in Phase II trial}

Despite negative outcomes in clinical trials for Alzheimer's disease of potential therapeutic agents that engage in the $A \beta$ generation process, a number of companies still continue to explore this space - note Alzheon ALZ-801 news in this article. Now Biogen and Eisai, partnering on the development of BAN2401, an anti-A $\beta$ protofibril antibody that binds to neutralize and eliminate soluble, toxic A $\beta$ aggregates, have updated on their Phase II study of this antibody. The trial, in patients with prodromal or mild Alzheimer's disease (collectively known as early Alzheimer's disease) and carrying specific biomarkers, assessed changes from baseline to 18 months, using biomarkers including amyloid accumulation in the brain (by amyloid positron emission tomography). Clinical end points assessments - Alzheimer's Disease Composite Score, Alzheimer's Disease Assessment Scale-cognitive subscale and Clinical Dementia Rating Sum of Boxes were also used. A dose-dependent statistically significant reduction in amyloid plaque as measured by amyloid positron emission tomography was seen at all doses, with at the highest doses having $81 \%$ of treated patients converted from amyloid positive to amyloid negative at 18 months. There was a dose-dependent slowing in cognitive decline at 18 months from baseline on Alzheimer's Disease Composite Score, Alzheimer's Disease Assessment Scale-cognitive subscale and Clinical Dementia Rating Sum of Boxes, and BAN2401 showed acceptable tolerability [47]. This outcome may vindicate the two companies' decision late last year to continue the trial, after an Independent Data Monitoring Committee determined that it did not meet the criteria for success based on a Bayesian analysis at 12 months. By using Bayesian statistical approach, they had hoped to demonstrate clinical success faster than more traditional study designs. The 18-month final study analysis represents the treatment time that may be more appropriate for assessing efficacy in disease-modifying agents [48]. The companies have stated that they will discuss the findings with the regulatory authorities to determine a path forward.

\section{Favorable Phase Ib data for PXL770 (Poxel SA) enables advance to Phase IIa in NASH}

Lyon, France-based Poxel SA, a biopharmaceutical company pursuing the development of innovative treatments for metabolic diseases, including NASH, has announced the outcome of a Phase I trials on PXL770. PXL770 is a first-in-class, oral, direct AMP-activated protein kinase activator being advanced for the treatment of NASH. The unique mechanism of action of PXL779 may have the potential to act on the underlying causes of fatty liver disease. AMP-activated protein kinase is involved in multiple metabolic pathways associated with the control of lipid 
metabolism and glucose homeostasis, hence is an attractive target in a wide range of metabolic diseases including $\mathrm{NASH}$, for which there are no current specific approved therapies, and the disease is increasing in prevalence.

Favorable pharmacokinetic and drug interaction data, as well as the safety and tolerability profile obtained in the Phase I studies have enabled planning for Phase IIa studies, which are expected to start in the second half of 2018 [49].

\section{Implantable device for delivery of wet age-related macular degeneration therapy ranibizumab (Lucentis ${ }^{\circledR}$, Genentech) shows favorable Phase II trial data}

A small refillable device, slightly longer than a grain of rice and designed for surgical implantation in the eye to continuously deliver ranibizumab over time, has been successfully evaluated in a Phase II study by Genentech (CA, USA), a company within the Roche group [50]. The device, the port delivery system (PDS), containing ranibizumab, has been studied in patients with wet age-related macular degeneration, a major cause of sight loss in people over 60. It is refillable in place, using a customized needle and which can be done in a physician's office. Currently, this disease can be treated with the anti-VEGF antibody ranibizumab by a monthly injection into the eye (Lucentis ${ }^{\circledR}$, Genentech), but constitutes a high treatment burden, which can lead to under-treatment, hence less than desirable outcomes. The majority of patients (approximately $60-80 \%$, depending on the dose) in the trial who had the device implanted were able to go for 6 months or longer before the device required refilling. The trial compared the efficacy and safety of the ranibizumab-filled PDS with monthly intravitreal injections of ranibizumab. Patients receiving the PDS ranibizumab $100 \mathrm{mg} / \mathrm{ml}$ achieved similar gains in visual acuity, and similar reductions in central retinal thickness to patients receiving monthly ranibizumab $0.5 \mathrm{mg}$. Phase III studies of the device/drug combination are expected to start later in 2018. The device was developed by ForSight VISION4, which was acquired by Roche in 2017 [51].

\section{Precancerous actinic keratosis cleared with topical KX2-391 (Athenex, Almirall)}

KX2-391 (KX-01), is a dual Src kinase/tubulin polymerization inhibitor that binds for both targets through a novel site, affecting tumor growth and metastases through Src, and cell division through action on tubulin polymerization. It has been investigated in Phase III trials as a $1 \%$ ointment for topical therapy for actinic keratosis, a common skin condition caused by UV light damage, which presents as patches of thick, scaly or crusty skin. It is the most common precancerous skin condition, which if not treated can progress to squamous cell carcinoma. In actinic keratosis of the face and scalp, ointment was applied once daily for 5 days, in two double-blind, vehicle placebo-controlled trials. The primary end point of $100 \%$ clearance by day 57 was achieved. Local skin reactions were mild and rapidly resolved [52]. KX2-391 was licensed by Athenex to Almirall, S A (Barcelona, Spain) late last year, with Almirall being granted rights to research, develop and commercialize KX2-391 in the USA, Europe and Russia, with agreed milestones that encourage the development of additional indications and formulations [53].

Pembrolizumab (Keytruda ${ }^{\circledR}$, Merck) monotherapy Phase III trials in head \& neck cancer

Potentially extending further the role of immuno-oncology as a first-line therapeutic option, Merck has shared the results of a Phase III trial of pembrolizumab (Keytruda ${ }^{\circledR}$ ) in recurrent or metastatic head and neck squamous cell carcinoma in patients with appropriate PD-L1 biomarker status. As monotherapy, pembrolizumab treatment resulted in significantly longer overall survival compared with the current standard of care in head and neck squamous cell carcinoma, cetuximab in combination with platinum chemotherapy plus 5-flourouracil [54]. Based on the result of the interim analysis, the trial will continue without modification, resulting regulatory filings at some future date.

\section{NGF inhibitor tanezumab (Pfizer/Lilly) for treatment of osteoarthritis severe pain}

New Phase III osteoarthritis clinical trial data from a Pfizer (NY, USA)/Lilly collaboration demonstrated that a novel, humanized monoclonal antibody NGF inhibitor delivered statistically significant improvement in pain, physical function and the patients' overall assessment of their disease compared with a placebo injection. NGF levels increase in the body in response to injury, inflammation or in chronic pain states, and an inhibitor like tanezumab is thought to interrupt the transmission of pain signals from peripheral tissues to the spinal cord and the brain. The results demonstrate the potential benefit of a nonopioid treatment for pain relief in these patients. The treatment was well tolerated, with a low frequency of rapidly progressive osteoarthritis and no occurrences of osteonecrosis, which had been reported in earlier trials of tanezumab but may possibly be associated with the concomitant use 
of NSAIDs in affected subjects. Tanezumab is also being explored in the treatment of chronic low back pain and cancer pain associated with bone metastases [55,56].

\section{Dolutegravir (ViiV Healthcare) in fixed combination two-drug regimens has similar outcome to established three-drug regimens}

ViiV Healthcare (London, UK) has shared results of studies investigating fixed combinations of dolutegravir, an HIV integrase inhibitor with a second antiretroviral agent. Standard of care for treatment of HIV infections to date has been based on triple drug combinations. A combination of dolutegravir with the NRTI lamivudine was shown to have similar efficacy at 48 weeks, as measured by numbers of copies of viral RNA in plasma, as a three-drug regimen combination of dolutegravir, tenofovir disoproxil fumarate (a prodrug NRTI) and the NRTI emtricitabine. ViiV is planning to seek regulatory approval for a fixed-dose combination of dolutegravir and lamivudine later in 2018 [57]. ViiV also announced 100-week Phase III clinical trial data for the two-drug fixed combination of dolutegravir with the second-generation NNRTI rilpivirine, again demonstrating the utility of a two-drug combination therapy approach. Adequately virologically suppressed patients switched from three or four-drug antiretroviral regimens, maintained the degree of virological RNA in plasma after switching [58]. ViiV presented both trials at the 22nd International AIDS Conference, held in Amsterdam, The Netherlands (23-27 July 2018). The trials indicate the possibility of reducing drug burden for HIV patients while maintaining the effectiveness of treatment. The fixed-dose combination of dolutegravir and rilpivirine (Juluca ${ }^{\circledR}$ ) was approved in the USA in late 2017 and in Europe, Canada and Australia in mid-2018, and is under review in other territories.

\section{Long-term immune response data in healthy volunteers for an investigational HIV-1 preventive vaccine (Janssen Pharmaceutical Companies)}

A robust HIV antibody response has been seen to be maintained 1 year after the last vaccination in all the healthy volunteers who participated in the Phase I/II study of an investigational, preventive vaccine against HIV-1 infection being developed by Janssen Pharmaceutical Companies (Beerse, Belgium), the company announced at the 22nd International AIDS Conference (Amsterdam, The Netherlands [23-27 July 2018]). The eventual elimination of HIV will require a preventative vaccine, though this is highly challenging, due to the nature of the virus including the genetic diversity of the virus across the world. Janssen's approach has been to create a mosaic-based vaccine, containing immunogens using genes taken from a variety of viral subtypes and so potentially able to prevent infections due to a wide range of strains of HIV-1. The first efficacy study is getting underway, in order to determine if the vaccine is safe and able to reduce HIV infection in a vulnerable population of young women in five countries across sub-Saharan Africa. First results for this trial are expected in 2021. Additional trials will follow [59].

\section{Financial \& competing interests disclosure}

The author is a former employee, and is a current shareholder, of Bristol-Myers Squibb, which has active research programs and marketed medicines in some of the therapeutic areas covered in this article and some of those products are mentioned by name. The author has consulting and advisory roles with Summit Therapeutics, F2G and Galecto Biotech.

The author has no other relevant affiliations or financial involvement with any organization or entity with a financial interest in or financial conflict with the subject matter or materials discussed in the manuscript apart from those disclosed.

No writing assistance was utilized in the production of this manuscript.

\section{References}

1. Timmins P. Industry update: the latest developments in the field of therapeutic delivery 1-28 February 2018. Ther. Deliv. 9(7), 493-514 (2018).

2. Vicore Pharma and HealthCap create a leading drug development company focused on severe lung diseases. http://vicorepharma.com/cision/?cisionId=F7E30E3EE199756A

3. Axovant announces global licensing agreement for AXO-AAV-OPMD program for treatment of oculopharyngeal muscular dystrophy and broader platform collaboration with Benitec Biopharma.

http://investors.axovant.com/news-releases/news-release-details/axovant-announces-global-licensing-agreement-axo-aav-opmd

4. Otsuka Pharmaceutical to acquire Visterra. www.otsuka.com/en/hd_release/release/pdf.php?news=1837

5. Proprietary Hierotope ${ }^{\circledR}$ platform. http://visterrainc.com/technology/hierotope-platform.html

6. BioCryst Pharmaceuticals announces termination of merger agreement with Idera Pharmaceuticals. http://ir.biocryst.com/news-releases/news-release-details/biocryst-pharmaceuticals-announces-termination-merger-agreement 
7. PTC Therapeutics to acquire Agilis Biotherapeutics.

http://ir.ptcbio.com/news-releases/news-release-details/ptc-therapeutics-acquire-agilis-biotherapeutics

8. Agilis DNA therapeutic programs. www.agilisbio.com/pipeline/dna-therapeutic-programs/

9. Genmab Enters strategic partnership with Immatics to discover and develop next generation bispecific cancer immunotherapies. http://ir.genmab.com/news-releases/news-release-details/genmab-enters-strategic-partnership-immatics-discover-and

10. Kite and Gadeta announce strategic collaboration to advance $\gamma \delta \mathrm{T}$ cell receptor technology for solid tumors. https://docs.wixstatic.com/ugd/7e6f33_ab43b8bcleef46deb1090c22f249208a.pdf

11. Roivant subsidiary Dermavant Sciences signs agreement with GSK to purchase rights to tapinarof. www.gsk.com/en-gb/media/press-rele ases/roivant-subsidiary-dermavant-sciences-signs-agreement-with-gsk-to-purchase-rights-to-tapinarof/

12. PureTech Health announces collaboration with Roche to advance technology for oral administration of antisense oligonucleotides. http://puretechhealth.com/news/23-press-releases/1029-puretech-health-announces-collaboration-with-roche-to-advance-technologyfor-oral-administration-of-antisense-oligonucleotides

13. Milk exosomes technology. http://puretechhealth.com/puretech-pipeline/our-pipeline/details/milk-exosomes-technology

14. Novartis demonstrates commitment to lead immuno-dermatology by in-licensing IL-17C compound for atopic dermatitis. www.novartis.com/news/media-releases/novartis-demonstrates-commitment-lead-immuno-dermatology-licensing-il-17c-compound-at opic-dermatitis

15. Discovery of an allosteric inhibitor of SHP2, RMC- 4550.

https://revmed.egnyte.com/dl/vOOAEE3CBV/4878_Discovery_of_an_allosteric_inhibitor_of_SHP2\%2C_RMC-4550.pdf_

16. Sanofi and REVOLUTION Medicines launch global partnership to develop potential first-in-class targeted treatments for multiple cancers. www.revolutionmedicines.com/media/sanofi-and-revolution-medicines-launch-global-partnership-develop-potential-first-class

17. Sangamo Therapeutics to acquire TxCell.

https://investor.sangamo.com/press-releases/detail/414/sangamo-therapeutics-to-acquire-txcell

18. Biogen Q2 2018 revenues increase $9 \%$ to $\$ 3.4$ billion. http://investors.biogen.com/static-files/44a04b54-7322-4009-8dfb-08cf7a79b80a

19. MedImmune and 4D Molecular Therapeutics collaborate to design, develop and commercialize AAV gene therapy for chronic lung disease. www.4dmoleculartherapeutics.com/medimmune-and-4d-molecular-therapeutics-collaborate-to-design-develop-and-commerci alize-aav-gene-therapy-for-chronic-lung-disease/

20. Bristol-Myers Squibb and Gritstone Oncology announce clinical research collaboration to evaluate novel immunotherapy approach in advanced solid tumors. https://gritstoneoncology.com/news-1/bristol-myers-squibb-and-gritstone-oncology-announce-clinical-researc h-collaboration-to-evaluate-novel-immunotherapy-approach-in-advanced-solid-tumors

21. Takeda and Ovid Therapeutics expand clinical program for TAK-935/OV935 with three new studies in rare developmental and epileptic encephalopathies (DEE). www.takeda.com/newsroom/newsreleases/2018/takeda-and-ovid-therapeutics-expand-clinical-progr am-for-tak-935ov935-with-three-new-studies-in-rare-developmental-and-epileptic-encephalopathies-dee/

22. Takeda and Ovid Therapeutics announce innovative clinical development and commercialization collaboration for TAK-935 in rare pediatric epilepsies. http://investors.ovidrx.com/phoenix.zhtml?c=254470\&p=irol-newsArticle\&ID=2239671

23. Anima Biotech announces an exclusive collaboration with Lilly for the discovery and development of translation inhibitors of several protein targets. https://www.animabiotech.com/sites/default/files/pr/anima-press-release-20180722.pdf

24. GSK and 23andMe sign agreement to leverage genetic insights for the development of novel medicines. www.gsk.com/en-gb/media/pres s-releases/gsk-and-23andme-sign-agreement-to-leverage-genetic-insights-for-the-development-of-novel-medicines/

25. Convelo Therapeutics to develop new regenerative medicines for neurological disease characterized by loss of myelin. www.convelotx.com/news/2018/7/24/convelo-therapeutics-to-develop-new-regenerative-medicines-for-neurological-disease-character ized-by-loss-of-myelin

26. Hubler Z, Allimuthu D, Bederman I et al. Accumulation of 8,9-unsaturated sterols drives oligodendrocyte formation and remyelination. Nature 560, 372-376 (2018).

27. Alector announces $\$ 133$ million in series $\mathrm{E}$ financing to advance broad portfolio of immuno-neurology and immuno-oncology programs. https://static1.squarespace.com/static/58d3fd42cd0f68ac6fe60833/t/5b5859308a922def01942d9a/1532516657043/Alector Series_E_7.25.2018.pdf

28. Inversago Pharma receives $\$ 7$ million in series A financing. https://inversago.com/en/news-and-events/

29. Alzheon presents new findings in APOE $4 / 4$ homozygous patients with mild Alzheimer's disease, further supporting precision medicine approach and planned confirmatory clinical trial with ALZ-801.

https://alzheon.com/alzheon-presents-new-findings-in-apoe4-4-homozygous-patients-with-mild-alzheimers-disease-further-supporti ng-precision-medicine-approach-and-planned-confirmatory-clinical-trial-with-alz-801/

30. ALZ-801. https://alzheon.com/alzheon-pipeline/amyloid-oligomers-ad/ 
31. Hey JA, Yu JY, Versavel M et al. Clinical pharmacokinetics and safety of ALZ-801, a novel prodrug of tramiprosate in development for the treatment of Alzheimer's disease. Clin. Pharmacokinet. 57(3), 315-333 (2018).

32. IFM Therapeutics, LLC launches subsidiary, IFM Tre, with $\$ 31$ million series A financing to develop NLRP3 antagonists for inflammatory diseases. https://www.if mthera.com/news/ifm-therapeutics-llc-launches-subsidiary-ifm-tre/

33. Bristol-Myers Squibb to acquire IFM Therapeutics to strengthen oncology pipeline focus on innate immunity. www.if mthera.com/new s/bristol-myers-squibb-to-acquire-if m-therapeutics-to-strengthen-oncology-pipeline-focus-on-innate-immunity/

34. FDA grants approval of TIBSOVO ${ }^{\circledR}$, the first oral, targeted therapy for adult patients with relapsed/refractory acute myeloid leukemia and an IDH1 mutation.

http://investor.agios.com/news-releases/news-release-details/fda-grants-approval-tibsovor-first-oral-targeted-therapy-adult

35. AbbVie receives US FDA approval of ORILISSA ${ }^{\text {TM }}$ (elagolix) for the management of moderate to severe pain associated with endometriosis. https://news.abbvie.com/news/press-releases/abbvie-receives-us-fda-approval-orilissa-elagolix-for-management-moderat e-to-severe-pain-associated-with-endometriosis.htm

36. Neurocrine Biosciences reports second quarter 2018 financial results.

http://phoenix.corporate-ir.net/phoenix.zhtml?c=68817\&p=RssLanding\&cat=news\&id=2361035

37. US Food and Drug Administration approves SIGA Technologies' TPOXX ${ }^{\circledR}$ (tecovirimat) for the treatment of smallpox. https://investor.siga.com/news-releases/news-release-details/us-food-and-drug-administration-approves-siga-technologies

38. US FDA approves Krintafel (tafenoquine) for the radical cure of $P$. vivax malaria. www.gsk.com/en-gb/media/press-releases/us-fda-approves-krintafel-tafenoquine-for-the-radical-cure-of-p-vivax-malaria/

39. Amgen and UCB provide update on regulatory status Of EVENITY ${ }^{\top M}$ (romosozumab) in the US. https://wwwext.amgen.com/en-gb/m edia/news-releases/2017/07/amgen-and-ucb-provide-update-on-regulatory-status-of-evenity-romosozumab-in-the-us/

40. UCB and Amgen resubmit biologics license application (BLA) for EVENITY ${ }^{\top M}$ (Romosozumab) to the US FDA. www.ucb.com/stories-media/Press-Releases/article/UCB-and-Amgen-Resubmit-Biologics-Licence-Application-BLA-for-EVENITYT M-Romosozumab-to-the-U-S-FDA

41. SCYNEXIS reports positive results from Phase $2 \mathrm{~b}$ dose-finding study of oral SCY-078 in vulvovaginal candidiasis. www.scynexis.com/news-media/press-releases/detail/155/scynexis-reports-positive-results-from-phase-2b

42. Zogenix announces positive top-line results from second pivotal Phase 3 clinical trial of ZX008 in Dravet syndrome. https://zogenixinc.gcs-web.com/news-releases/news-release-details/zogenix-announces-positive-top-line-results-second-pivotal-phase

43. Zogenix announces positive top-line results from pivotal Phase 3 clinical trial of ZX008 in Dravet syndrome. https://zogenixinc.gcs-web.com/news-releases/news-release-details/zogenix-announces-positive-top-line-results-pivotal-phase-3

44. PureTech Health affiliate resTORbio announces positive topline results in Phase $2 \mathrm{~b}$ trial of RTB101. http://puretechhealth.com/news/23 -press-releases/1030-puretech-health-affiliate-restorbio-announces-positive-topline-results-in-phase-2b-trial-of-rtb101

45. Cassiopea announces very positive top-line Phase 3 results for Winlevi ${ }^{\circledR}$ (Clascoterone) cream in treating acne. www.cassiopea.com/news-and-media/press-releases/yr-2018/180710.aspx

46. Cassiopea announces very positive interim analysis Phase 2 results for Breezula ${ }^{\circledR}$ (Clascoterone) in treating androgenetic alopecia. www.cassiopea.com/news-and-media/press-releases/yr-2018/180716.aspx

47. Eisai and Biogen announce detailed results of Phase II clinical study of BAN2401 in early Alzheimer's disease at Alzheimer's Association International Conference (AAIC) 2018.

http://investors.biogen.com/news-releases/news-release-details/eisai-and-biogen-announce-detailed-results-phase-ii-clinical

48. Adaptive Phase II study of BAN2401 in early Alzheimer's disease continues toward 18-month endpoint. http://investors.biogen.com/news-releases/news-release-details/adaptive-phase-ii-study-ban2401-early-alzheimers-disease

49. Poxel announces favorable results for PXL770 Phase $1 \mathrm{~b}$ multiple ascending dose trial and drug-drug interaction study. www.poxelpharma.com/en_us/news-media/press-releases/detail/97/poxel-announces-favorable-results-for-pxl770-phase- $1 \mathrm{~b}$

50. Genentech unveils positive Phase II results for the first-ever eye implant to achieve sustained delivery of a biologic medicine to treat people with wet age-related macular degeneration (AMD).

https://www.gene.com/media/press-releases/14739/2018-07-25/genentech-unveils-positive-phase-ii-resu

51. ForSight VISION4, Inc. announces acquisition by Roche www.prnewswire.com/news-releases/forsight-vision4-inc-announces-acquisition-by-roche-300388249.html

52. Athenex announces that two Phase 3 pivotal efficacy studies of KX2-391 in actinic keratosis achieved their primary endpoints. http://ir.athenex.com/phoenix.zhtml?c=254495\&p=irol-newsArticle\&ID=2360203

53. Almirall and Athenex announce strategic partnership for the treatment of actinic keratosis. www.almirall.com $/ \mathrm{en} / \mathrm{media} / \mathrm{press}$-releases $/ \mathrm{m}$ edia-detail-new?title=almirall-and-athenex-announce-strategic-partnership-for-the-treatment-of-actinic-keratos- 1 \&articleId=2132320

54. KEYTRUDA ${ }^{\circledR}$ (pembrolizumab) monotherapy met a primary endpoint in the Phase 3 KEYNOTE-048 trial, significantly improving OS as first-line therapy in head and neck squamous cell carcinoma patients whose tumors expressed PD-L1 (CPS $\geq 20)$. www.mrknewsr oom.com/news-release/oncology/keytruda-pembrolizumab-monotherapy-met-primary-endpoint-phase-3-keynote-048-tr 
55. Pfizer and Lilly announce positive top-line results from Phase 3 trial of tanezumab for the treatment of osteoarthritis (OA) pain. www.pfizer.com/news/press-release/press-release-detail/pfizer_and_lilly_announce_positive_top_line_results_from_phase_3_trial_of_tanez umab_for_the_treatment_of_osteoarthritis_oa_pain

56. Hochberg MC. Serious joint-related adverse events in randomized controlled trials of anti-nerve growth factor monoclonal antibodies. Osteoarthr. Cartil. 23(S1), S18-S21 (2015).

57. ViiV Healthcare presents Phase III data at AIDS 2018 from landmark GEMINI studies showing two-drug regimen of dolutegravir and lamivudine has similar efficacy to a three-drug regimen in treatment naive HIV patients, with no emergence of resistance. www.gsk.com/en-gb/media/press-releases/viiv-healthcare-presents-phase-iii-data-at-aids-2018-from-landmark-gemini-studies/

58. ViiV Healthcare announces SWORD 100-week data for Juluca (dolutegravir/rilpivirine) at AIDS 2018. www.gsk.com/en-gb/media/pr ess-releases/viiv-healthcare-announces-sword-100-week-data-for-juluca-dolutegravirrilpivirine-at-aids-2018/

59. First long-term immune response data for investigational HIV-1 preventive vaccine announced by Johnson \& Johnson. www.janssen.com/first-long-term-immune-response-data-investigational-hiv-1-preventive-vaccine-announced-johnson-0 
